\title{
An Improved Expression for Information Quality of Basic Probability Assignment and Its Application in Fault Diagnosis
}

\author{
HANWEN LI ${ }^{1}$, RUI CAI ${ }^{2}$ \\ ${ }^{1}$ School of Computer and Information Science, Southwest University, Chongqing, 400715, China \\ ${ }^{2}$ School of Business College, Southwest University, Chongqing, 402460, China \\ Corresponding author: Rui Cai (e-mail: cairuiswu@163.com).
}

This work was supported in part by the Fundamental Research Funds for the Central Universities under Grant XDJK2019C085, and in part by the Chongqing Overseas Scholars Innovation Program under Grant cx2018077.

\begin{abstract}
Information quality is widely used in many applications. However, how to measure information quality in basic probability assignment accurately is still an open issue. Generalized expression for information quality is an effective method to measure information quality in basic probability assignment. Nevertheless, the counter-intuitive results may be obtained when statements are of intersection. To address this issue, a new expression for information quality of basic probability assignment is proposed in this paper considering the frame of discernment and the influence of intersection among statements which can cause changes of uncertainty. Numerical examples are illustrated to demonstrate the effectiveness of the proposed method. In addition, an application in fault diagnosis is used to show the effectiveness of the proposed method.
\end{abstract}

INDEX TERMS Information quality, Gini entropy, Dempster-Shafer evidence theory, Basic probability assignment, Fault diagnosis

\section{INTRODUCTION}

The world is pervaded with uncertainties, and decisions must be made based on uncertain information. DempsterShafer evidence theory is an easy and effective framework for modeling uncertain information [1]-[5]. It is widely used in uncertainty reasoning and it has the ability to process many types of information in the real world to make accurate decision. There are also many other mathematical models to do uncertainty modeling like D numbers [6]-[10], Z numbers [11]-[16], fuzzy sets [17]-[20], intuitionistic fuzzy sets [21][25], pythagorean fuzzy sets [26]-[29], two-dimensional belief function [30], intuitionistic evidence sets [31], and so on.

Information quality is firstly proposed by Yager and Petry based on Gini entropy to measure the uncertainty for a probability distribution [32], [33]. It has been widely used in pattern classification [34], [35], decision making [36], [37] and so on [38]-[43], [43], [44]. Li et al. propose a generalized expression for information quality in basic probability assignment in Dempster-Shafer evidence theory [45], which makes information quality have greater scope of application.

Yager and Petry's method does not consider the length of each element and treat elements that have different length equally, which is counter-intuitive. Li et al.'s method takes the length of each element into account, and fully considers the potential uncertainty created by non-single elements. However, when elements are of intersection, the previous methods do not take the effects of intersection of statements into account, counter-intuitive results may be obtained. To address this issue, an improved expression for information quality is proposed considering the length of frame of discernment(FOD) and the influence of intersection among statements. An exponential item is added to take the effects of intersection into account. The proposed expression can degenerate into generalized form of information quality and information quality proposed by Yager and Petry under certain conditions.

The rest of this paper is organised as follows. Section II introduces the preliminaries include Dempster-Shafer theory, information quality and generalized form of information quality. Improved expression for information quality in basic probability assignment will be proposed in Section III. In Section IV, numerical examples will be given to illustrate the effectiveness of proposed method. In Section V, an application in fault diagnosis is given to show the effectiveness 
of proposed method. Finally, conclusions will be made in Section VI

\section{PRELIMINARIES}

In this section, the preliminaries will be briefly introduced, including Dempster-Shafer evidence theory and information quality.

\section{A. DEMPSTER-SHAFER EVIDENCE THEORY}

Dempster-Shafer evidence theory can represent the uncertainties effectively, and it is widely used in many fields like pattern classification [46]-[48], multi-criteria decision making [49]-[51] and so on [52]-[61]. The brief introduction to Dempster-Shafer evidence theory will be given as follows. Definition 2.1: A set of hypothesis $\Theta$ is called the frame of discernment. It is defined as follows:

$$
\Theta=\left\{h_{1}, h_{2}, \ldots, h_{n}\right\}
$$

The power set $2^{\Theta}$ is defined as:

$$
2^{\Theta}=\left\{\emptyset,\left\{h_{1}\right\},\left\{h_{1}, h_{2}\right\}, \ldots,\left\{h_{1}, h_{2}, \ldots, h_{n}\right\}\right\}
$$

where $\emptyset$ is an empty set.

Definition 2.2: The discernment frame $\Theta$ must satisfy the following conditions:

(1) $m(\emptyset)=0$

(2) $\sum_{A \in \Theta} m(A)=1$

where $m: 2^{\Theta} \rightarrow[0,1]$ is called the basic probability assignment(BPA). And $m$ is also known as the mass function. For an BPA $m$ on $\Theta$, each subset $A$ of $\Theta$ is called an focal element of $m$ if $m(A)>0$.

How to fuse different information is still an open issue. The basic combination rule is given as follows.

Definition 2.3: Suppose there are two independent BPAs, $m_{1}, m_{2}$, The combination rule is used to fuse two BPAs. And the result of fused BPA is denoted by $m$. And it is given by:

$$
\left\{\begin{array}{l}
m(\emptyset)=0 \\
m(A)=\frac{\sum_{B \cap C=A} m_{1}(B) m_{2}(C)}{1-K}
\end{array}\right.
$$

where $K=\sum_{B \cap C=\emptyset} m_{1}(B) m_{2}(C)$.

The counter-intuitive results may be obtained when information is highly conflicted [62]. Hence, many methods are proposed to address this issue [63]-[67]. The weighted average method is proposed by Deng et al. based on Murphy's average method and Jousselme distance [68]-[70].

Definition 2.4: Given two mass functions $m_{1}$ and $m_{2}$, the Jousselme distance between $m_{1}$ and $m_{2}$ is defined as:

$$
d\left(m_{1}, m_{2}\right)=\sqrt{\frac{1}{2}\left(\overrightarrow{m_{1}}-\overrightarrow{m_{2}}\right)^{T} \underline{\underline{D}}\left(\overrightarrow{m_{1}}-\overrightarrow{m_{2}}\right)}
$$

where $\overrightarrow{m_{1}}$ and $\overrightarrow{m_{2}}$ are respective belief functions in the notation of vector(and each size is $2^{\Theta}-1$ ). And $\underline{D}$ is an $\left|2^{\Theta}\right| \times\left|2^{\Theta}\right|$ matrix that each of its element is $\underline{\underline{D}}\left(s_{1}, s_{2}\right)=$ $\frac{s_{1} \cap s_{2}}{s_{1} \cup s_{2}} \mid . s_{1}, s_{2} \in 2^{\Theta}$.
The similarity measure between $m_{1}$ and $m_{2}$ can be defined as

$$
\operatorname{Sim}\left(m_{1}, m_{2}\right)=1-d\left(m_{1}, m_{2}\right)
$$

Suppose there are $n$ mass functions. And we can construct a $2^{n} \times 2^{n}$ similarity matrix as follows:

$$
A=\left[\begin{array}{cccc}
1 & S_{12} & \ldots & S_{1 n} \\
S_{21} & 1 & \ldots & S_{2 n} \\
\vdots & \vdots & \vdots & \vdots \\
S_{n 1} & S_{n 2} & \ldots & 1
\end{array}\right]_{n \times n}
$$

And $S_{i j}=\operatorname{Sim}\left(m_{i}, m_{j}\right)$, for $i=1,2, \ldots, n, j=1,2, \ldots, n$.

The support degree of one mass function $m_{i}(i=$ $1,2, \ldots, n)$ can be defined as:

$$
\operatorname{Sup}\left(m_{i}\right)=\sum_{j=1}^{n} S_{i j}
$$

And the credibility degree of of one mass function $m_{i}(i=$ $1,2, \ldots, n)$ can be denoted as:

$$
\operatorname{Crd}_{i}=\frac{\operatorname{Sup}\left(m_{i}\right)}{\sum_{j=1}^{n} \operatorname{Sup}\left(m_{j}\right)}
$$

And the weighted mass function can be obtained by weighted average as follows:

$$
\begin{gathered}
w_{i}=\operatorname{Crd}_{i} \\
m(A)=\sum_{i=1}^{n} w_{i} m_{i}(A)
\end{gathered}
$$

where $A$ is a focal element of $m$. Then weighted mass function can be used to fuse data in the later process.

\section{B. INFORMATION QUALITY}

Entropy is a measurement of the uncertainty of information. The larger the value of entropy, the bigger the uncertainty of information. Many methods for entropy can represent the uncertainty for information, like Shannon entropy [71], Tsallis entropy [72]-[74], Rényi entropy [75], [76], Deng entropy [77]-[88], generalized belief entropy [89] and so on [90]-[92].

Based on Gini entropy [33], information quality is proposed by Yager and Petry as another way to measure the degree of uncertainty of information [32]. The value of information quality is larger while the uncertainty of information is smaller. Information quality is defined as follows.

Definition 2.5: Given a probability function $p_{i}$, the information quality of $p_{i}$ is defined as:

$$
I Q_{p_{i}}=\sum_{j=1}^{n}\left|p_{i j}\right|^{2}
$$

When $p_{i j}=1$, the defined information quality reaches its maximum value, and when all $p_{i j}=\frac{1}{n}$, which leads to most uncertainty of information, the value of information quality is smallest. 
Inspired by the idea of Deng entropy [77], Li et al. propose generalized form of information quality in basic probability assignment [45], and it is defined as follows.

Definition 2.6: Given a basic probability allocation $m_{i}$, the generalized information quality of $m_{i}$ is defined as follows.

$$
I Q_{m_{i}}=\sum_{A \in X}\left(\frac{m_{i}(A)}{2^{|A|}-1}\right)^{2}
$$

When all the statements are all single elements, the generalized form of information quality will degenerate into the form of information quality proposed by Yager and Petry.

\section{PROPOSED METHOD}

In this paper, we focus on the disadvantage of generalized form of information quality on the ignorance of the intersection between statements. In this section, we propose a new form of information quality based on generated form of information quality. The proposed form of information quality considering the length of FOD and the intersection between statements is given as follows.

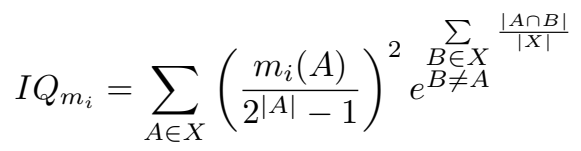

Where $|A|$ denotes the cardinality of proposition $A$, and $|A \cap B|$ denotes the cardinality of the intersection between proposition $A$ and $B .|X|$ denotes the length of FOD. When all propositions have no intersection, the proposed form of information quality degenerates into Eq.(11). Then if belief is only assigned to single elements, the proposed form will degenerate into Eq.(10).

\section{NUMERICAL EXAMPLES}

In this section, numerical examples will be given to demonstrate the efficiency of the proposed form of information quality.

Example 4.1: Suppose there are two basic probability allocations $m_{1}$ and $m_{2}$. The frame of discernment $X=$ $\{a, b, c, d\} \cdot m_{1}(a, b)=0.5, m_{1}(b, c)=0.5, m_{2}(a, b)=$ $0.5, m_{2}(c, d)=0.5$.

Intuitively, the information quality of $m_{1}$ is larger than $m_{2}$ because focal elements of $m_{1}$ are in intersection. Although the distributions of two mass functions are similar, $m_{1}$ has less information volume than $m_{2}$ as $m_{1}$ has less targets than $m_{2}$. Hence, $m_{1}$ contains less information than $m_{2}$ and the value of information quality of $m_{1}$ is larger than $m_{2}$.

With Yager and Petry's method [32], the information quality of $m_{1}$ and $m_{2}$ can be calculated as

$$
\begin{aligned}
I Q_{m_{1}} & =0.5^{2}+0.5^{2} \\
\log \left(I Q_{m_{1}}\right) & =-1.3863 \\
I Q_{m_{2}} & =0.5^{2}+0.5^{2} \\
\log \left(I Q_{m_{2}}\right) & =-1.3863
\end{aligned}
$$

With the generalized form of information quality [45], the information quality of $m_{1}$ and $m_{2}$ can be calculated as

$$
\begin{aligned}
I Q_{m_{1}} & =\left(\frac{0.5}{2^{2}-1}\right)^{2}+\left(\frac{0.5}{2^{2}-1}\right)^{2} \\
\log \left(I Q_{m_{1}}\right) & =-2.8904 \\
I Q_{m_{2}} & =\left(\frac{0.5}{2^{2}-1}\right)^{2}+\left(\frac{0.5}{2^{2}-1}\right)^{2} \\
\log \left(I Q_{m_{2}}\right) & =-2.8904
\end{aligned}
$$

With the proposed method, the information quality can be calculated as

$$
\begin{aligned}
I Q_{m_{1}} & =\left(\frac{0.5}{2^{2}-1}\right)^{2} e^{\frac{1}{4}}+\left(\frac{0.5}{2^{2}-1}\right)^{2} e^{\frac{1}{4}} \\
\log \left(I Q_{m_{1}}\right) & =-2.6404 \\
I Q_{m_{2}} & =\left(\frac{0.5}{2^{2}-1}\right)^{2} e^{\frac{0}{4}}+\left(\frac{0.5}{2^{2}-1}\right)^{2} e^{\frac{0}{4}} \\
\log \left(I Q_{m_{2}}\right) & =-2.8904
\end{aligned}
$$

As we can see, information qualities of $m_{1}$ and $m_{2}$ are the same calculated by Yager and Petry method and Li et al.'s method. Counter-intuitive results are obtained. With the proposed method, information quality of $m_{1}$ is larger than $m_{2}$, which is intuitive and reasonable. In this example, proposed method has better performance than existed methods.

Example 4.2: Suppose there are three basic probability allocations $m_{1}, m_{2}$ and $m_{3}$. The frame of discernment $X=\{a, b, c, d, e, f\} \cdot m_{1}(a, b)=0.2, m_{1}(c, d)=$ $0.6, m_{1}(e, f)=0.2, m_{2}(a, b)=0.2, m_{2}(b, c)=0.6$, $m_{2}(c, f)=0.2, m_{3}(a, b)=0.2, m_{3}(b, c)=0.6$, $m_{3}(e, f)=0.2$.

Intuitively, the information qualities of $m_{1}, m_{2}$ and $m_{3}$ are not the same. The information quality of $m_{1}$ is lowest while the information quality of $m_{2}$ is highest as elements in $m_{2}$ have largest intersection.

With Yager and Petry's method [32], the information quality can be obtained as follows.

$$
\begin{aligned}
I Q_{m_{1}} & =0.2^{2}+0.6^{2}+0.2^{2} \\
\log \left(I Q_{m_{1}}\right) & =-0.8210 \\
I Q_{m_{2}} & =0.2^{2}+0.6^{2}+0.2^{2} \\
\log \left(I Q_{m_{2}}\right) & =-0.8210 \\
I Q_{m_{3}} & =0.2^{2}+0.6^{2}+0.2^{2} \\
\log \left(I Q_{m_{3}}\right) & =-0.8210
\end{aligned}
$$

With Li et al.'s method [45], the information quality can be obtained as follows.

$$
\begin{aligned}
I Q_{m_{1}} & =\left(\frac{0.2}{2^{2}-1}\right)^{2}+\left(\frac{0.6}{2^{2}-1}\right)^{2}+\left(\frac{0.2}{2^{2}-1}\right)^{2} \\
\log \left(I Q_{m_{1}}\right) & =-3.0182 \\
I Q_{m_{2}} & =\left(\frac{0.2}{2^{2}-1}\right)^{2}+\left(\frac{0.6}{2^{2}-1}\right)^{2}+\left(\frac{0.2}{2^{2}-1}\right)^{2} \\
\log \left(I Q_{m_{2}}\right) & =-3.0182
\end{aligned}
$$




$$
\begin{aligned}
I Q_{m_{3}} & =\left(\frac{0.2}{2^{2}-1}\right)^{2}+\left(\frac{0.6}{2^{2}-1}\right)^{2}+\left(\frac{0.2}{2^{2}-1}\right)^{2} \\
\log \left(I Q_{m_{3}}\right) & =-3.0182
\end{aligned}
$$

With proposed method, the information quality can be calculated as follows.

$$
\begin{aligned}
I Q_{m_{1}} & =\left(\frac{0.2}{2^{2}-1}\right)^{2} e^{\frac{0}{6}}+\left(\frac{0.6}{2^{2}-1}\right)^{2} e^{\frac{0}{6}} \\
& +\left(\frac{0.2}{2^{2}-1}\right)^{2} e^{\frac{0}{6}} \\
\log \left(I Q_{m_{1}}\right) & =-3.0182 \\
I Q_{m_{2}} & =\left(\frac{0.2}{2^{2}-1}\right)^{2} e^{\frac{1}{6}}+\left(\frac{0.6}{2^{2}-1}\right)^{2} e^{\frac{2}{6}} \\
& +\left(\frac{0.2}{2^{2}-1}\right)^{2} e^{\frac{1}{6}} \\
\log \left(I Q_{m_{2}}\right) & =-2.7132 \\
I Q_{m_{3}} & =\left(\frac{0.2}{2^{2}-1}\right)^{2} e^{\frac{1}{6}}+\left(\frac{0.6}{2^{2}-1}\right)^{2} e^{\frac{1}{6}} \\
& +\left(\frac{0.2}{2^{2}-1}\right)^{2} e^{\frac{0}{6}} \\
\log \left(I Q_{m_{3}}\right) & =-2.8656
\end{aligned}
$$

As we can see, information qualities of three mass functions are the same calculated by Yager and Petry method and Li et al.'s method. The obtained results are counterintuitive. With the proposed method, information quality of $m_{2}$ is largest and information quality of $m_{1}$ is smallest. It is intuitive and reasonable.

Example 4.3: Suppose there is a basic probability allocation $m$, the FOD is $\Theta=\{1,2, \ldots, 14\} . m(6)=0.05$, $m(3,4,5)=0.05, m(\Theta)=0.8, m(A)=0.1, A$ ranges from $\{1\}$ to $\{1,2, \ldots, 14\}$.

Figure 1 demonstrates the change of logarithmic value of different measures for information quality when $A$ changes. Table 1 shows the logarithmic value of different measures for information quality in different cases. Values of Yager and Petry's method keep almost stable when A changes because the length of A do not affect the result, and it is not reasonable. Values calculated by proposed method is larger than Li et al.'s method, and it is reasonable because the effect of intersection is taken into account, and information quality is larger. Values calculated by two methods have same trends of change.

Example 4.4: Suppose there is a basic probability allocation $m$, the FOD is $\Theta=\{1,2, \ldots, 14\} . m(1)=0.05, m(2)=$ $0.05, \ldots, m(14)=0.05, m(A)=0.05, A$ ranges from $\{1\}$ to $\{1,2, \ldots, 14\}$.

Figure 2 demonstrates the change of logarithmic value of different measures for information quality when $A$ changes. Table 2 shows the logarithmic value of information quality calculated by proposed method in different cases. When the size of A becomes larger, information qualities calculated by Eq.(10) and Eq.(11) approach to a certain value. Because of

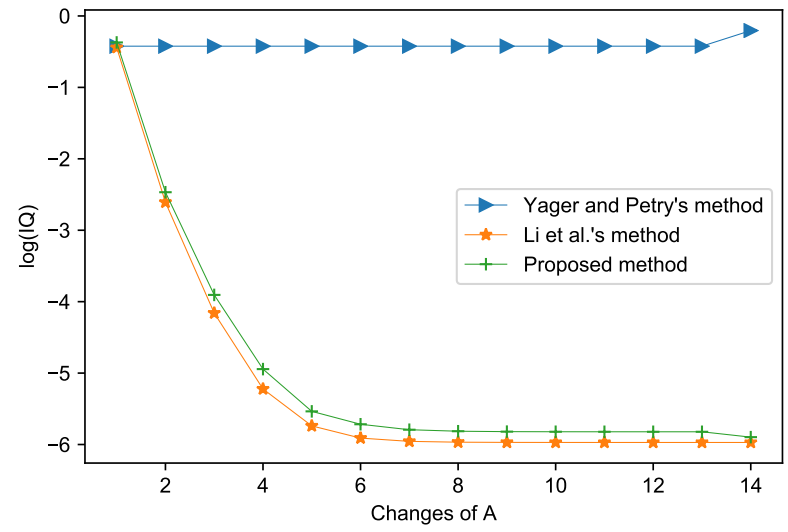

FIGURE 1: Different measurements of information quality with changes of A of BPA in Example 4.3

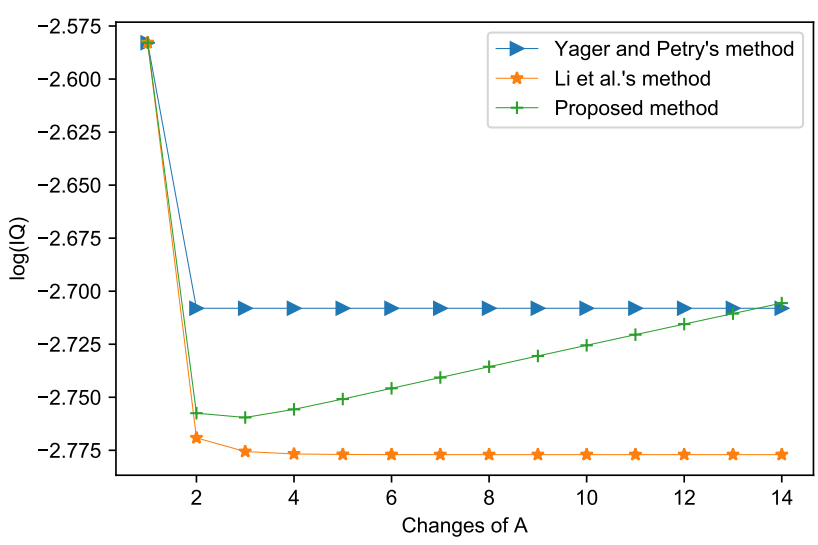

FIGURE 2: Different measurements of information quality with changes of A of BPA in Example 4.4

the effect of intersection elements, the information quality calculated by the proposed method is larger than it calculated by Eq.(10) and smaller than it calculated by Eq.(11) at most of times. It is reasonable that elements have more mutual information with changes of $A$, which leads to the increment of information quality.

TABLE 1: Logarithmic value of different measurements for information quality with changes of A of BPA

\begin{tabular}{cccc}
\hline Cases & Yager's method [32] & Li et al's method [45] & Proposed method \\
\hline $\mathrm{A}=\{1\}$ & -0.4231 & -0.4423 & -0.3709 \\
$\mathrm{~A}=\{1,2\}$ & -0.4231 & -2.6083 & -2.4677 \\
$\mathrm{~A}=\{1,2,3\}$ & -0.4231 & -4.1597 & -3.9054 \\
$\mathrm{~A}=\{1,2,3,4\}$ & -0.4231 & -5.2222 & -4.9442 \\
$\mathrm{~A}=\{1,2, \ldots, 5\}$ & -0.4231 & -5.7393 & -5.5359 \\
$\mathrm{~A}=\{1,2, \ldots, 6\}$ & -0.4231 & -5.9100 & -5.7164 \\
$\mathrm{~A}=\{1,2, \ldots, 7\}$ & -0.4231 & -5.9558 & -5.7928 \\
$\mathrm{~A}=\{1,2, \ldots, 8\}$ & -0.4231 & -5.9674 & -5.8140 \\
$\mathrm{~A}=\{1,2, \ldots, 9\}$ & -0.4231 & -5.9703 & -5.8197 \\
$\mathrm{~A}=\{1,2, \ldots, 10\}$ & -0.4231 & -5.9710 & -5.8212 \\
$\mathrm{~A}=\{1,2, \ldots, 11\}$ & -0.4231 & -5.9712 & -5.8217 \\
$\mathrm{~A}=\{1,2, \ldots, 12\}$ & -0.4231 & -5.9712 & -5.8218 \\
$\mathrm{~A}=\{1,2, \ldots, 13\}$ & -0.4231 & -5.9713 & -5.8218 \\
$\mathrm{~A}=\{1,2, \ldots, 14\}$ & -0.2046 & -5.9713 & -5.8968 \\
\hline
\end{tabular}


TABLE 2: Logarithmic value of information quality with changes of A of BPA

\begin{tabular}{cc}
\hline Cases & Logarithmic value \\
\hline $\mathrm{A}=\{1\}$ & -2.5829 \\
$\mathrm{~A}=\{1,2\}$ & -2.7575 \\
$\mathrm{~A}=\{1,2,3\}$ & -2.7595 \\
$\mathrm{~A}=\{1,2,3,4\}$ & -2.7560 \\
$\mathrm{~A}=\{1,2, \ldots, 5\}$ & -2.7508 \\
$\mathrm{~A}=\{1,2, \ldots, 6\}$ & -2.7458 \\
$\mathrm{~A}=\{1,2, \ldots, 7\}$ & -2.7407 \\
$\mathrm{~A}=\{1,2, \ldots, 8\}$ & -2.7356 \\
$\mathrm{~A}=\{1,2, \ldots, 9\}$ & -2.7305 \\
$\mathrm{~A}=\{1,2, \ldots, 10\}$ & -2.7255 \\
$\mathrm{~A}=\{1,2, \ldots, 11\}$ & -2.7205 \\
$\mathrm{~A}=\{1,2, \ldots, 12\}$ & -2.7155 \\
$\mathrm{~A}=\{1,2, \ldots, 13\}$ & -2.7106 \\
$\mathrm{~A}=\{1,2, \ldots, 14\}$ & -2.7056 \\
\hline
\end{tabular}

\section{APPLICATION}

In this section, an application in fault diagnosis is investigated using proposed expression for information quality. The case study in [93] is recalled in this section. Based on method proposed by Yuan et al. [94], a new method using improved expression for information quality is proposed and the main steps of this method are shown in Figure 3.

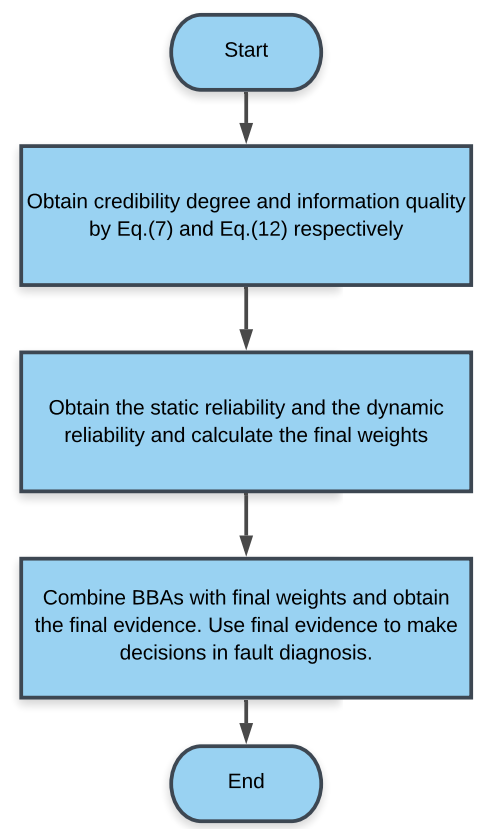

FIGURE 3: The flowchart of new method

In the example in [93], three fault types are called $F_{1}, F_{2}$ and $F_{3}$. The hypothesis set of faults is $\Theta=\left\{F_{1}, F_{2}, F_{3}\right\}$. Three sensors in the hypothesis set are independent. The results of fault diagnosis are called BOEs, denoted as $E_{1}, E_{2}$ and $E_{3}$. The BPAs of diagnosis results are shown in Table 3.

With the Dempster's combination rule in Eq.(3), the fused result is $m\left(F_{1}\right)=0.4519, m\left(F_{2}\right)=0.5048, m\left(F_{2}, F_{3}\right)=$ $0.0336, m(\Theta)=0.0096$. It is hard to judge which fault has been occurred because the values obtained by Dempster's combination rule are very close. The extreme values in $m_{2}$ influence the fused result.

To solve this problem, in [94], a fault diagnosis method is proposed. The reliability of each sensor is defined as the weight of a BOE. The weight of each BOE is defined as the product of a static reliability and a dynamic reliability, and it is defined as follows.

$$
w(i)=w_{s}(i) \times w_{d}(i)
$$

Where the static reliability $w_{s}\left(E_{1}\right)=1, w_{s}\left(E_{2}\right)=0.2040$, $w_{s}\left(E_{3}\right)=1$, in this example. The dynamic reliability is defined with the use of information quality as follows.

$$
w_{d}(i)=\operatorname{Crd}\left(m_{i}\right) \times \frac{\frac{1}{I Q\left(m_{i}\right)}}{\max \left(\frac{1}{I Q\left(m_{j}\right)}\right)}, j=1,2,3
$$

In this example, the credibility degree and information quality can be obtained by Eq.(7) and Eq.(12). The values of them are:

$$
\begin{aligned}
\operatorname{Crd}\left(m_{1}\right) & =1 \\
\operatorname{Crd}\left(m_{2}\right) & =0.5523 \\
\operatorname{Crd}\left(m_{3}\right) & =0.9660 \\
I Q_{m_{1}} & =0.5280 \\
I Q_{m_{2}} & =1.2116 \\
I Q_{m_{3}} & =0.7071
\end{aligned}
$$

The weight of each BOE can be calculated.

$$
\begin{aligned}
w(1) & =w_{s}(1) \times \operatorname{Crd}\left(m_{1}\right) \times \frac{\frac{1}{I Q\left(m_{1}\right)}}{\frac{1}{I Q\left(m_{1}\right)}} \\
& =1 \times 1 \times \frac{\frac{1}{0.5280}}{\frac{1}{0.5280}}=0.5778 \\
w(2) & =w_{s}(2) \times \operatorname{Crd}\left(m_{2}\right) \times \frac{\frac{1}{I Q\left(m_{2}\right)}}{\frac{1}{I Q\left(m_{1}\right)}} \\
& =0.2040 \times 0.5523 \times \frac{\frac{1}{1.2116}}{\frac{1}{0.5280}}=0.0274 \\
w(3) & =w_{s}(3) \times \operatorname{Crd}\left(m_{3}\right) \times \frac{\frac{1}{I Q\left(m_{3}\right)}}{\frac{1}{I Q\left(m_{1}\right)}} \\
& =1 \times 0.9660 \times \frac{1}{\frac{1}{0.5280}}=0.3947
\end{aligned}
$$

The final fused result can be obtained by Eq.(3) and Eq.(9). $m\left(F_{1}\right)=0.8996, m\left(F_{2}\right)=0.0685, m\left(F_{2}, F_{3}\right)=0.0245$ $m(\Theta)=0.0074$. It is easy to tell and conclude that $F_{1}$ is the fault with the highest probability. The results with different methods are shown in Table 4. As Table 4 shows, the proposed method is compatible with Fan et al.'s method, Yuan et al.'s method and Zhou et al.'s method.

The application in fault diagnosis shows the effectiveness of the proposed form of information quality. Also, this application indicates a promising application prospect of this new form of information quality. 
TABLE 3: BPAs of diagnosis result

\begin{tabular}{c|c|c|c|c}
\hline Sensor report & $F_{1}$ & $F_{2}$ & $F_{2}, F_{3}$ & $\Theta$ \\
\hline$E_{1}: m_{1}$ & 0.60 & 0.10 & 0.20 & 0.10 \\
\hline$E_{2}: m_{2}$ & 0.05 & 0.80 & 0.05 & 0.10 \\
\hline$E_{3}: m_{3}$ & 0.70 & 0.10 & 0.10 & 0.10 \\
\hline
\end{tabular}

TABLE 4: Fault diagnosis results with different methods

\begin{tabular}{c|c|c|c|c}
\hline Method & $m\left(F_{1}\right)$ & $m\left(F_{2}\right)$ & $m\left(F_{2}, F_{3}\right)$ & $m(\Theta)$ \\
\hline Dempster's combination rule & 0.4519 & 0.5048 & 0.0336 & 0.0096 \\
\hline Fan et al.'s method [93] & 0.8119 & 0.1096 & 0.0526 & 0.0259 \\
\hline Yuan et al.'s method [94] & 0.8948 & 0.0739 & 0.0241 & 0.0072 \\
\hline Zhou et al.'s method [81] & 0.8951 & 0.0738 & 0.0240 & 0.0071 \\
\hline Proposed method & 0.8996 & 0.0685 & 0.0245 & 0.0074 \\
\hline
\end{tabular}

\section{CONCLUSION}

In this paper, an improved expression for information quality is proposed considering the scale of the frame of discernment and the intersection between statements in basic probability of assignment, based on generalized form of information quality. Moreover, some numerical examples are illustrated to show the effectiveness of the proposed method. Results show that proposed method has better performance than the previous methods. In addition, an application in fault diagnosis is used to illustrate the effectiveness of the proposed method. In the future, we will further explore on other applications of this new form of information quality in basic probability assignment.

\section{ACKNOWLEDGMENT}

The authors declare that there are no conflicts of interest.

\section{REFERENCES}

[1] A. P. Dempster, "Upper and Lower Probabilities Induced by a MultiValued Mapping," Annals of Mathematical Statistics, vol. 38, no. 2, pp. 325-339, 1967.

[2] G. Shafer, A Mathematical Theory of Evidence, 01 1976, vol. 1.

[3] Y. Deng, "Generalized evidence theory," Applied Intelligence, vol. 43, no. 3, pp. 530-543, 2015.

[4] F. Xiao, "Generalization of Dempster-Shafer theory: A complex mass function," Applied Intelligence, 2019.

[5] R. R. Yager, "Generalized dempster-shafer structures," IEEE Transactions on Fuzzy Systems, vol. 27, no. 3, pp. 428-435, 2018.

[6] Y. Deng, "D numbers: theory and applications," Journal of Information \&Computational Science, vol. 9, no. 9, pp. 2421-2428, 2012.

[7] X. Deng, Y. Hu, Y. Deng, and S. Mahadevan, "Supplier selection using AHP methodology extended by D numbers," Expert Systems with Applications, vol. 41, no. 1, pp. 156-167, 2014.

[8] X. Deng and Y. Deng, "D-AHP method with different credibility of information," Soft Computing, vol. 23, no. 2, pp. 683-691, 2019.

[9] F. Xiao, "A multiple-criteria decision-making method based on D numbers and belief entropy," International Journal of Fuzzy Systems, vol. 21, no. 4, pp. 1144-1153, 2019.

[10] X. Zhou, X. Deng, Y. Deng, and S. Mahadevan, "Dependence assessment in human reliability analysis based on D numbers and AHP," Nuclear Engineering and Design, vol. 313, pp. 243-252, 2017.

[11] L. A. Zadeh, "A note on Z-numbers," Information Sciences, vol. 181, no. 14, pp. 2923-2932, 2011.

[12] W. Jiang, Y. Cao, and X. Deng, "A Novel Z-network Model Based on Bayesian Network and Z-number," IEEE Transactions on Fuzzy Systems, 2019.

[13] B. Kang, D. Wei, Y. Li, and Y. Deng, "Decision making using Z-numbers under uncertain environment," Journal of computational Information systems, vol. 8, no. 7, pp. 2807-2814, 2012.
[14] B. Kang, Y. Deng, K. Hewage, and R. Sadiq, "A method of measuring uncertainty for Z-number," IEEE Transactions on Fuzzy Systems, vol. 27, no. 4, pp. 731-738, 2018.

[15] Q. Liu, Y. Tian, and B. Kang, "Derive knowledge of Z-number from the perspective of Dempster-Shafer evidence theory," Engineering Applications of Artificial Intelligence, vol. 85, pp. 754-764, 2019.

[16] B. Kang, Y. Deng, and R. Sadiq, "Total utility of Z-number," Applied Intelligence, vol. 48, no. 3, pp. 703-729, 2018.

[17] L. A. Zadeh, "Fuzzy sets," Information and control, vol. 8, no. 3, pp. 338353, 1965.

[18] C. V. Negoiţă and D. A. Ralescu, Applications of fuzzy sets to systems analysis. Springer, 1975.

[19] L. A. Zadeh, "Fuzzy sets and information granularity," Advances in fuzzy set theory and applications, vol. 11, pp. 3-18, 1979.

[20] F. Xiao, Z. Zhang, and J. Abawajy, "Workflow scheduling in distributed systems under fuzzy environment," Journal of Intelligent \& Fuzzy Systems, vol. 37, no. 4, pp. 5323-5333, 2019 .

[21] K. T. Atanassov, "Intuitionistic fuzzy sets," in Intuitionistic fuzzy sets. Springer, 1999, pp. 1-137.

[22] K. Atanassov, "New Operations Defined Over the Intuitionistic Fuzzy Sets," Fuzzy Sets and Systems, vol. 61, pp. 137-142, 011994.

[23] L. Dymova and P. Sevastjanov, "An interpretation of intuitionistic fuzzy sets in terms of evidence theory: Decision making aspect," KnowledgeBased Systems, vol. 23, no. 8, pp. 772-782, 2010.

[24] F. Xiao, "A distance measure for intuitionistic fuzzy sets and its application to pattern classification problems," IEEE Transactions on Systems, Man, and Cybernetics: Systems, 2019.

[25] C. Cheng, F. Xiao, and Z. Cao, "A new distance for intuitionistic fuzzy sets based on similarity matrix," IEEE Access, vol. 7, pp. 70 436-70 446, 2019.

[26] R. Yager and A. Abbasov, "Pythagorean Membership Grades, Complex Numbers, and Decision Making," International Journal of Intelligent Systems, vol. 28, pp. 436-452, 2013.

[27] R. Yager, "Pythagorean Membership Grades in Multicriteria Decision Making," IEEE Transactions on Fuzzy Systems, vol. 22, pp. 958-965, 2014.

[28] R. R. Yager, "Properties and applications of Pythagorean fuzzy sets," in Imprecision and Uncertainty in Information Representation and Processing. Springer, 2016, pp. 119-136.

[29] Q. Zhou, H. Mo, and Y. Deng, "A New Divergence Measure of Pythagorean Fuzzy Sets Based on Belief Function and Its Application in Medical Diagnosis," Mathematics, vol. 8, no. 1, p. 142, 2020.

[30] Y. Li and Y. Deng, "TDBF: Two-dimensional belief function," International Journal of Intelligent Systems, 2019.

[31] Y. Li and Y. Deng, "Intuitionistic evidence sets," IEEE Access, vol. 7, pp 106 417-106 426, 2019

[32] R. R. Yager and F. Petry, "An intelligent quality-based approach to fusing multi-source probabilistic information," Information Fusion, vol. 31, pp. 127-136, 2016

[33] C. Gini, E. Pizetti, and T. Salvemini, "Reprinted in Memorie di metodologica statistica," Rome: Libreria Eredi Virgilio Veschi, vol. 1, 1912.

[34] Z. Liu, Y. Liu, J. Dezert, and F. Cuzzolin, "Evidence combination based on credal belief redistribution for pattern classification," IEEE Transactions on Fuzzy Systems, 2019.

[35] Y. Song, X. Wang, L. Lei, and A. Xue, "A novel similarity measure on intuitionistic fuzzy sets with its applications," Applied Intelligence, vol. 42, no. 2, pp. 252-261, 2015.

[36] M. Song, W. Jiang, C. Xie, and D. Zhou, "A new interval numbers power average operator in multiple attribute decision making," International Journal of Intelligent Systems, vol. 32, no. 6, pp. 631-644, 2017.

[37] Z.-G. Liu, Q. Pan, J. Dezert, and A. Martin, "Combination of classifier with optimal weight based on evidential reasoning," IEEE Transactions on Fuzzy Systems, vol. 26, no. 3, pp. 1217-1230, 2017.

[38] T. T. Nguyen, T. C. Phan, Q. V. H. Nguyen, K. Aberer, and B. Stantic, "Maximal fusion of facts on the web with credibility guarantee," Information Fusion, vol. 48, pp. 55-66, 2019.

[39] H. Zhang and Y. Deng, "Engine fault diagnosis based on sensor data fusion considering information quality and evidence theory," Advances in Mechanical Engineering, vol. 10, no. 11, p. 1687814018809184, 2018.

[40] X. Cao and Y. Deng, "A new geometric mean FMEA method based on information quality," IEEE Access, vol. 7, pp. 95 547-95 554, 2019.

[41] D. Li and Y. Deng, "A New Correlation Coefficient Based on Generalized Information Quality," IEEE Access, vol. 7, pp. 175 411-175 419, 2019. 
[42] Y. Li and F. Xiao, "Bayesian Update with Information Quality under the Framework of Evidence Theory," Entropy, vol. 21, no. 1, p. 5, 2019.

[43] S. Liang, X. Deng, and W. Jiang, "Optimal data fusion based on information quality function," Applied Intelligence, pp. 1-9, 2019.

[44] Y. Li and F. Xiao, "Aggregation of uncertainty data based on ordered weighting aggregation and generalized information quality," International Journal of Intelligent Systems, 2019.

[45] D. Li, X. Gao, and Y. Deng, "A Generalized Expression for Information Quality of Basic Probability Assignment," IEEE Access, vol. 7, pp. 174 734-174739, 2019.

[46] E. Mandler and J. Schümann, "Combining the classification results of independent classifiers based on the Dempster/Shafer theory of evidence," in Machine Intelligence and Pattern Recognition. Elsevier, 1988, vol. 7, pp. 381-393.

[47] T. Denoeux, "Analysis of evidence-theoretic decision rules for pattern classification," Pattern recognition, vol. 30, no. 7, pp. 1095-1107, 1997.

[48] T. Denœux and L. M. Zouhal, "Handling possibilistic labels in pattern classification using evidential reasoning," Fuzzy sets and systems, vol. 122, no. 3, pp. 409-424, 2001.

[49] M. Beynon, D. Cosker, and D. Marshall, "An expert system for multicriteria decision making using Dempster Shafer theory," Expert Systems with Applications, vol. 20, no. 4, pp. 357-367, 2001

[50] Y. Deng, F. T. Chan, Y. Wu, and D. Wang, "A new linguistic MCDM method based on multiple-criterion data fusion," Expert Systems with Applications, vol. 38, no. 6, pp. 6985-6993, 2011.

[51] F. Xiao, "EFMCDM: Evidential fuzzy multicriteria decision making based on belief entropy," IEEE Transactions on Fuzzy Systems, 2019.

[52] J.-B. Yang and D.-L. Xu, "On the evidential reasoning algorithm for multiple attribute decision analysis under uncertainty," IEEE Transactions on Systems, Man, and Cybernetics-Part A: Systems and Humans, vol. 32, no. 3, pp. 289-304, 2002 .

[53] J.-B. Yang and M. G. Singh, "An evidential reasoning approach for multiple-attribute decision making with uncertainty," IEEE Transactions on systems, Man, and Cybernetics, vol. 24, no. 1, pp. 1-18, 1994.

[54] Y.-M. Wang, J.-B. Yang, and D.-L. Xu, "Environmental impact assessment using the evidential reasoning approach," European Journal of Operational Research, vol. 174, no. 3, pp. 1885-1913, 2006.

[55] J.-B. Yang, "Rule and utility based evidential reasoning approach for multiattribute decision analysis under uncertainties," European journal of operational research, vol. 131, no. 1, pp. 31-61, 2001.

[56] L. Sun, R. P. Srivastava, and T. J. Mock, "An information systems security risk assessment model under the Dempster-Shafer theory of belief functions," Journal of Management Information Systems, vol. 22, no. 4, pp. 109-142, 2006.

[57] P. Dutta, "Uncertainty modeling in risk assessment based on DempsterShafer theory of evidence with generalized fuzzy focal elements," Fuzzy information and engineering, vol. 7, no. 1, pp. 15-30, 2015.

[58] J. Yang, H.-Z. Huang, L.-P. He, S.-P. Zhu, and D. Wen, "Risk evaluation in failure mode and effects analysis of aircraft turbine rotor blades using Dempster-Shafer evidence theory under uncertainty," Engineering Failure Analysis, vol. 18, no. 8, pp. 2084-2092, 2011.

[59] J. Wang, Y. Hu, F. Xiao, X. Deng, and Y. Deng, "A novel method to use fuzzy soft sets in decision making based on ambiguity measure and Dempster-Shafer theory of evidence: an application in medical diagnosis," Artificial intelligence in medicine, vol. 69, pp. 1-11, 2016.

[60] Q. Dong and X. Liu, "Risk assessment of water security in Haihe River Basin during drought periods based on D-S evidence theory," Water Science and Engineering, vol. 7, no. 2, pp. 119 - 132, 2014.

[61] H. Wang, X. Deng, Z. Zhang, and W. Jiang, "A New Failure Mode and Effects Analysis Method Based on Dempster-Shafer Theory by Integrating Evidential Network," IEEE Access, vol. 7, pp. 79 579-79 591, 2019.

[62] L. A. Zadeh, "A Simple View of the Dempster-Shafer Theory of Evidence and Its Implication for the Rule of Combination," Ai Magazine, vol. 7, no. 2, pp. 85-90, 1986

[63] E. Lefevre, O. Colot, and P. Vannoorenberghe, "Belief function combination and conflict management," Information Fusion, vol. 3, no. 2, pp. 149 $-162,2002$

[64] W. Liu, "Analyzing the degree of conflict among belief functions," Artificial Intelligence, vol. 170, no. 11, pp. 909-924, 2006.

[65] F. Xiao, "A new divergence measure for belief functions in D-S evidence theory for multisensor data fusion," Information Sciences, vol. 514, pp. $462-483,2020$.

[66] W. Zhang and Y. Deng, "Combining conflicting evidence using the DEMATEL method,” Soft computing, vol. 23, no. 17, pp. 8207-8216, 2019.
[67] Z. Wang and F. Xiao, "An improved multisensor data fusion method and its application in fault diagnosis," IEEE Access, vol. 7, pp. 3928-3937, 2018

[68] C. K. Murphy, "Combining belief functions when evidence conflicts," Decision Support Systems, vol. 29, no. 1, pp. 1 - 9, 2000.

[69] A.-L. Jousselme, D. Grenier, and Íęloi Bossíę, "A new distance between two bodies of evidence," Information Fusion, vol. 2, no. 2, pp. 91 - 101, 2001.

[70] D. Yong, S. WenKang, Z. ZhenFu, and L. Qi, "Combining belief functions based on distance of evidence," Decision Support Systems, vol. 38, no. 3 pp. $489-493,2004$.

[71] C. E. Shannon, "A mathematical theory of communication," Bell system technical journal, vol. 27, no. 3, pp. 379-423, 1948.

[72] C. Tsallis, "Possible generalization of Boltzmann-Gibbs statistics," Journal of statistical physics, vol. 52, no. 1-2, pp. 479-487, 1988.

[73] X. Gao, F. Liu, L. Pan, Y. Deng, and S.-B. Tsai, "Uncertainty measure based on Tsallis entropy in evidence theory," International Journal of Intelligent Systems, vol. 34, no. 11, pp. 3105-3120, 2019.

[74] T. Wen and W. Jiang, "Measuring the complexity of complex network by Tsallis entropy," Physica A: Statistical Mechanics and its Applications, vol. 526, p. 121054, 2019.

[75] A. Rényi et al., "On measures of entropy and information," in Proceedings of the Fourth Berkeley Symposium on Mathematical Statistics and Probability, Volume 1: Contributions to the Theory of Statistics. The Regents of the University of California, 1961.

[76] S. Duan, T. Wen, and W. Jiang, "A new information dimension of complex network based on Rényi entropy," Physica A: Statistical Mechanics and its Applications, vol. 516, pp. 529-542, 2019.

[77] Y. Deng, "Deng entropy," Chaos, Solitons \& Fractals, vol. 91, pp. 549553, 2016.

[78] B. Kang and Y. Deng, "The maximum Deng entropy," IEEE Access, vol. 7 , pp. $120758-120765,2019$.

[79] J. Abellán, "Analyzing properties of Deng entropy in the theory of evidence," Chaos, Solitons \& Fractals, vol. 95, pp. 195-199, 2017.

[80] L. Pan and Y. Deng, "A new belief entropy to measure uncertainty of basic probability assignments based on belief function and plausibility function," Entropy, vol. 20, no. 11, p. 842, 2018.

[81] D. Zhou, Y. Tang, and W. Jiang, "A modified belief entropy in DempsterShafer framework," PloS one, vol. 12, no. 5, p. e0176832, 2017.

[82] H. Cui, Q. Liu, J. Zhang, and B. Kang, "An improved deng entropy and its application in pattern recognition," IEEE Access, vol. 7, pp. 18284 $18292,2019$.

[83] D. Wang, J. Gao, and D. Wei, "A new belief entropy based on Deng entropy," Entropy, vol. 21, no. 10, p. 987, 2019.

[84] Y. Zhao, D. Ji, X. Yang, L. Fei, and C. Zhai, "An Improved Belief Entropy to Measure Uncertainty of Basic Probability Assignments Based on Deng Entropy and Belief Interval," Entropy, vol. 21, no. 11, p. 1122, 2019.

[85] Y. Tang, D. Zhou, and F. T. Chan, "An extension to Deng's entropy in the open world assumption with an application in sensor data fusion," Sensors, vol. 18, no. 6, p. 1902, 2018.

[86] Y. Tang, D. Zhou, S. Xu, and Z. He, "A weighted belief entropy-based uncertainty measure for multi-sensor data fusion," Sensors, vol. 17, no. 4 p. $928,2017$.

[87] M. N. Khan and S. Anwar, "Paradox Elimination in Dempster-Shafer Combination Rule with Novel Entropy Function: Application in DecisionLevel Multi-Sensor Fusion,” Sensors, vol. 19, no. 21, p. 4810, 2019.

[88] D. Zhou, Y. Tang, and W. Jiang, "An improved belief entropy and its application in decision-making," Complexity, vol. 2017, 2017.

[89] F. Liu, X. Gao, J. Zhao, and Y. Deng, "Generalized belief entropy and its application in identifying conflict evidence," IEEE Access, vol. 7, pp. $126625-126633,2019$.

[90] P. Garrido, S. Goldstein, and J. L. Lebowitz, "Boltzmann entropy for dense fluids not in local equilibrium," Physical Review Letters, vol. 92, no. 5, p 050602, 2004.

[91] G. Benettin, L. Galgani, and J.-M. Strelcyn, "Kolmogorov entropy and numerical experiments," Physical Review A, vol. 14, no. 6, p. 2338, 1976

[92] C. Tsallis, "Nonadditive entropy: the concept and its use," The European Physical Journal A, vol. 40, no. 3, p. 257, 2009.

[93] X. Fan and M. J. Zuo, "Fault diagnosis of machines based on D-S evidence theory. Part 1: D-S evidence theory and its improvement," Pattern Recognition Letters, vol. 27, no. 5, pp. 366-376, 2006.

[94] K. Yuan, F. Xiao, L. Fei, B. Kang, and Y. Deng, "Modeling sensor reliability in fault diagnosis based on evidence theory," Sensors, vol. 16, no. 1 , p. 113,2016 


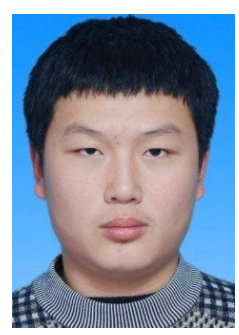

HANWEN LI is currently a Sophomore Studen$t$ and pursuing the bachelor's degree from the School of Computer and Information Science, Southwest University, China. His research interests include information fusion, social computing, complex networks, graph neural networks and graph mining.

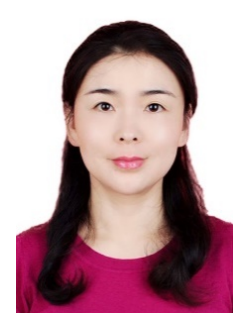

RUI CAl received the Ph.D degree in Management Science and Engineering from Chongqing University, Chongqing, China. in 2018. She is an Associate Professor in the School of business college, Southwest University, Chongqing, China. Her research interests include project management and decision making. 